\title{
Sublittoral soft bottom communities and diversity of Mejillones Bay in northern Chile (Humboldt Current upwelling system)
}

\author{
Jürgen Laudien • Manuel E. Rojo • Marcelo E. Oliva • \\ Wolf E. Arntz $\cdot$ Sven Thatje
}

Received: 23 June 2006 / Revised: 5 January 2007 / Accepted: 8 January 2007 / Published online: 21 March 2007

(C) Springer-Verlag and AWI 2007

\begin{abstract}
The macrozoobenthos of Mejillones Bay $\left(23^{\circ} \mathrm{S}\right.$; Humboldt Current) was quantitatively investigated over a 7-year period from austral summer 1995/1996 to winter 2002. About 78 van Veen grab samples taken at six stations (5, 10, $20 \mathrm{~m}$ depth) provided the basis for the analysis of the distribution of 60 species and 28 families of benthic invertebrates, as well as of their abundance and biomass. Mean abundance (2,119 individuals $\mathrm{m}^{-2}$ ) was in the same order compared to a previous investigation; mean biomass (966 $\mathrm{g}$ formalin wet mass $\mathrm{m}^{-2}$ ), however, exceeded prior estimations mainly due to the dominance of the bivalve Aulacomya ater. About $43 \%$ of the taxa inhabited the complete depth range. Mean taxonomic Shannon diversity $\left(\mathrm{H}^{\prime}\right.$, Log e) was $1.54 \pm 0.58$ with a maximum at $20 \mathrm{~m}$ $(1.95 \pm 0.33)$; evenness increased with depth. The fauna was numerically dominated by carnivorous gastropods, polychaetes and crustaceans (48\%). About $15 \%$ of the species were suspensivorous, $13 \%$ sedimentivorous, $11 \%$ detritivorous, $7 \%$ omnivorous and $6 \%$ herbivorous. Cluster
\end{abstract}

Communicated by H.-D. Franke.

J. Laudien $(\varangle) \cdot$ W. E. Arntz

Alfred Wegener Institute for Polar and Marine Research,

P.O. Box 120161, 27515 Bremerhaven, Germany

e-mail: Juergen.Laudien@awi.de

M. E. Rojo · M. E. Oliva

Facultad de Recursos del Mar,

Universidad de Antofagasta,

Casilla 170, Antofagasta, Chile

S. Thatje

National Oceanography Centre, Southampton,

School of Ocean and Earth Science,

University of Southampton, European Way,

SO14 3ZH Southampton, UK analyses showed a significant difference between the shallow and the deeper stations. Gammarid amphipods and the polychaete family Nephtyidae characterized the 5-mzone, the molluscs Aulacomya ater, Mitrella unifasciata and gammarids the intermediate zone, while the gastropod Nassarius gayi and the polychaete family Nereidae were most prominent at the deeper stations. The communities of the three depth zones did not appear to be limited by hypoxia during non-El Niño conditions. Therefore, no typical change in community structure occurred during El Niño 1997-1998, in contrast to what was observed for deeper faunal assemblages and hypoxic bays elsewhere in the coastal Humboldt Current system.

Keywords Macrozoobenthos · Community analyses · Feeding modes $\cdot$ Mejillones Bay $\cdot$ El Niño

\section{Introduction}

Eastern Boundary Currents both of the Pacific and the Atlantic Ocean fuel the circular oceanic current systems, transporting cold water into tropical and equatorial regions. Due to wind-driven upwelling with a return of nutrients to the euphotic zone, these ecosystems are very productive (Barber and Smith 1981). The Humboldt Current is recognized as one of the largest and in terms of secondary production most productive marine ecosystems (Mann and Lazier 1991). In its northern branch coastal upwelling is restricted to upwelling cells within a very narrow continental shelf off central and northern Chile. Several studies have shown the existence of cold upwelled water in nearshore zones (e.g. Fonseca 1989; Escribano 1998; Marín et al. 2001; Giraldo et al. 2002), sustaining large biomasses of small pelagic fish (Alheit and Bernal 1993). 
The northern Chilean Peninsula Mejillones $\left(23^{\circ} \mathrm{S}\right.$, $70^{\circ} \mathrm{W}$; Antofagasta area) encloses two opposing bays: the southward orientated Bay of Antofagasta and the northward orientated Bay of Mejillones, which is situated in a transition zone between shallow northward-flowing Subantarctic Waters and the surface manifestation of the poleward undercurrent consisting of southward-flowing Equatorial Waters. This oceanographic pattern can be affected by the large-scale El Niño Southern Oscillation (ENSO) (e.g. Taraba 1959; Brandhorst 1971; Navea and Miranda 1980; Fonseca 1989; Arntz and Fahrbach 1991; Fuenzalida 1992a, b; Morales et al. 1996; Gonzalez and Marín 1998; Strub et al. 1998; Escribano 1998; Ulloa et al. 2001; Arntz et al. 2006). Monitoring the sea surface temperature off Chile and Peru has illustrated that ENSO shows an interannual climate oscillation between warm phases (El Niño: EN) and enhanced cold phases (La Niña: LN). These two states of the ecosystem emerge from a background of "normal" conditions (e.g. McPhaden 1999).

Mejillones Bay is a well-described wind-driven nearshore upwelling site located near Punta Angamos (Fig. 1), one of the most productive cells along the northern Chilean coast (Escribano 1998; Marín and Olivares 1999; Escribano and Hidalgo 2000). Upwelling occurs year-round with a maximum and minimum intensity in October and January, respectively (e.g. Robles et al. 1975; Navea and Miranda 1980; Rodríguez et al. 1991; Marín et al. 1993, 2001; Escribano 1998; Marín and Olivares 1999; Pagès et al. 2001; Escribano et al. 2002). High primary production rates are sustained throughout the year, however the phytoplank- ton distribution may be highly patchy (Escribano 1998). Chlorophyll- $a$ concentrations of 10 (November 1983; Rodríguez et al. 1986) and $14 \mathrm{mg} \mathrm{m}^{-3}$ (January 2000; Giraldo et al. 2002) were measured at the surface of Mejillones Bay.

The high primary production is reflected in a pronounced benthic secondary production, which is 4.5 times higher in the coastal strip (0-20 m depth) compared to a depth of 20 $60 \mathrm{~m}$, and 60 times higher in relation to areas between 60 and $120 \mathrm{~m}$ (Ramorino and Muñiz 1970). At greater depths biomass values are negligible due to an extended zone in which the oxygen concentration is equal or lower than $0.5 \mathrm{ml} \mathrm{l}^{-1}$ (oxygen minimum zone-OMZ; Ramorino and Muñiz 1970; Levin 2003; Gallardo et al. 2004; Escribano et al. 2004). The oxygen minimum layer is typically shallow in the coastal area off northern Chile (Morales et al. 1996; Escribano et al. 2002, 2004) with an upper boundary, e.g. at $42 \mathrm{~m}$ during the austral summer 2000 (Giraldo et al. 2002) and between 20 and $40 \mathrm{~m}$ during spring 1996 (Escribano and Hidalgo 2000). Due to the lack of oxygen here invertebrate biomass is very low $\left(0.17 \mathrm{~g} \mathrm{~m}^{-2}\right.$ wet mass; Gallardo 1963; Neira et al. 2001; Arntz et al. 2006). Thus, only the narrow coastal fringe is subject to a strong artisanal fishery on benthic invertebrates (for Mejillones Bay, see Avendaño 1993). Previous studies focused on economically important invertebrate resources (e.g. Miranda 1975; Santa Cruz 1976; Gutiérrez and Zúñiga 1976; Henriquez and Olivares 1980; Follegati 1986; Avendaño and Cantillánez 1992; Chávez and Riquelme 1994; Avendaño and Le Pennec 1997; Moraga et al. 2001). Information on
Fig. 1 Map showing the study area located northeast of the village of Mejillones; sampling stations are indicated. The upper inlet includes a circulation scheme for the South Pacific coastal upwelling system off Chile (PSSCC Peru Sub-Surface Counter Current, $H C$ Humboldt Current, PCUC Peru-Chile Under Current; modified from Tarazona and Arntz 2001)

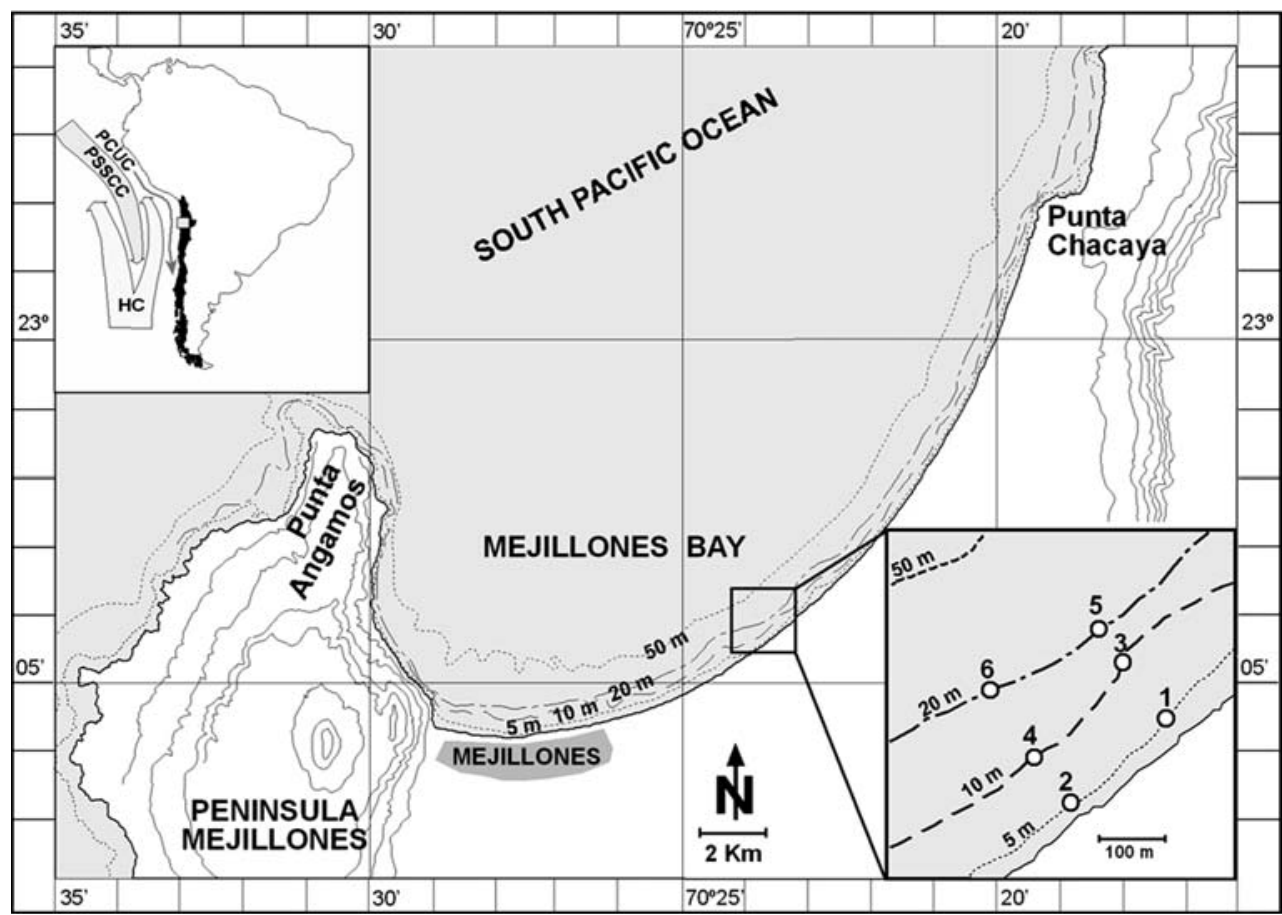


sublittoral benthic invertebrate communities off northern Chile is scarce and concentrates on a general description or deeper waters (20-18 ${ }^{\circ}$ : Gallardo 1963; Mejillones Bay: Ramorino and Muñiz 1970; Zúñiga et al. 1983; Hebbeln et al. 2001; Hebbeln and Wefer 2003; Palma et al. 2005). Shallower areas were subject of a naturalistic study describing the observations of SCUBA divers on community changes during and after El Niño 1982-1983 in a rocky substrate at Peninsula Mejillones (e.g. Tomicic 1985).

The study by Zúñiga et al. (1983) was the most comprehensive zoobenthic work covering the entire Mejillones Bay. However, the community was described cumulating all stations from 3 to $130 \mathrm{~m}$ depth. The local resources of high commercial interest are the mussel Aulacomya ater ("cholga"), the scallop Argopecten purpuratus ("ostión"), the sea urchin Loxechinus albus ("erizo") and the crab Cancer setosus ("jaiba"). Other fishing targets are several molluscs and crustaceans (e.g. Sernapesca 1995-2004).

The present study provides a quantitative description of the soft bottom macrozoobenthic community of Mejillones Bay, encompassing a time series of 7 years including EN 1997-1998. Three different depth zones of a shallow sublittoral soft-sediment habitat were chosen for comparison. The fauna was analysed for taxonomic composition, biomass and diversity as well as for feeding modes of dominant species. Variations in faunal associations are detected by cluster analysis from abundance data and are discussed in the context of EN.

\section{Materials and methods}

\section{Study area}

Mejillones Bay is located north of Antofagasta and northeast of Peninsula Mejillones in the central region of the coastal Humboldt Current upwelling system (Fig. 1). The bay covers about $285 \mathrm{~km}^{2}$ and has a maximum depth of $130 \mathrm{~m}$. About $11 \%$ of the bay do not exceed $20 \mathrm{~m}$ depth (Ramorino and Muñiz 1970). The area is subject to subequal semidiurnal tides with a maximum tide range of $1.7 \mathrm{~m}$; spring tides average $1.2 \mathrm{~m}$ and neap tides $0.4 \mathrm{~m}$. However, tidal currents are of less importance as the surface circulation is well correlated to wind driven upwelling (Marín et al. 2001). The seasonal cycle of the water column temperature is characterized by a cold mixed period in austral winter followed by a period of stratification during austral spring and summer, with a shallow thermocline (Escribano 1998). Mean sea surface temperature is $13.5^{\circ} \mathrm{C}$ in winter and rises up to $22^{\circ} \mathrm{C}$ in summer, while sediment temperature is $14^{\circ} \mathrm{C}$ at $3-20 \mathrm{~m}$ (Ramorino and Muñiz 1970) and ranges at $0-60 \mathrm{~m}$ between 12.7 and $15.7^{\circ} \mathrm{C}$ (Zúñiga et al. 1983). Salinity varies between 34.5 and 35.3 with a mean of $34.8( \pm 0.3)$ (Zúñiga et al. 1983; Rodríguez et al. 1986).

The present study area was located $5.5 \mathrm{~km}$ east of the village Mejillones and situated at the central south-eastern fringe of the bay $\left(23^{\circ} 04^{\prime} \mathrm{S}, 70^{\circ} 23^{\prime} \mathrm{W}\right.$ ) (Fig. 1). Here, the soft bottom inclines gradually from the coast. Sediments are composed of a fine sand-silt mixture (Ramorino and Muñiz 1970; Zúñiga et al. 1983; Valdés et al. 2005) with a thin oxygenated layer at 10 and $15 \mathrm{~m}(\sim 2 \mathrm{~cm}$, personal observation). A detailed description of the physical oceanography of Mejillones Bay was presented by Escribano et al. (2002, 2004). Chemical parameters and upwelling was subject of a study of Rodríguez et al. $(1991,1986)$ to which we refer.

\section{Macrofauna analyses}

Sampling was carried out twice a year along two station transects and at three different depths $(5,10,20 \mathrm{~m})$ (Fig. 1) covering the entire phytal, which ends at the $20 \mathrm{~m}$ isobath (Ramorino and Muñiz 1970). To analyse macrozoobenthos, at each station bottom samples were taken using a van Veen grab $\left(0.1 \mathrm{~m}^{2}\right)$ from austral summer 1995/1996 until austral winter 2002, except for winter 1996 (in total 13 sampling dates with six samples, each corresponding to a total area of $7.8 \mathrm{~m}^{2}$ ), and sieved through $1 \mathrm{~mm}$ mesh. All remaining material was preserved in $4 \%$ borax-buffered formalin. Thereafter, all macrofaunal organisms were sorted using a binocular microscope, identified and counted. Biomass was estimated from a preserved sub-sample by weighing after blotting on filter paper; valves of shelled organisms were opened for blotting. Percentages of animals in relation to total faunal abundances were calculated for the three different depth zones separately. Taxonomic Shannon-Wiener diversity indices $\left(\mathrm{H}^{\prime}\right.$, Log e; sensu Tarazona et al. 1996) were calculated for abundance values of each sample. Multivariate analysis was applied using the PRIMER $v 5$ package (Clarke and Gorley 2001). Data were square root transformed and Bray-Curtis similarities calculated. Classification (using group average linking) of samples was performed and groups of samples were distinguished based on the resultant dendrogram. Statistical differences were analysed by means of analysis of similarity (one-way ANOSIM, 95\% confidence interval; Clarke and Gorley 2001). Species with the highest frequency $(>75 \%)$ and significant dominance $(>5 \%)$ within a group were identified as characteristic of that group using SIMPER (Clarke and Gorley 2001).

Oceanographic and sedimentological conditions

In order to link environmental parameters with the biological results of this study, the granulometric structure of the sediment was classified. Hence, sediment samples were taken accordingly. The total grain size fraction was 
separated by dry sieving into six fractions: $<63 \mu \mathrm{m}=\operatorname{silt}(\Phi$ : $>4), 63-125 \mu \mathrm{m}=$ very fine sand $(\Phi: 4-3), 125-250 \mu \mathrm{m}=$ fine sand $(\Phi: 3-2), 250-500 \mu \mathrm{m}=$ medium sand $(\Phi: 2-1)$, $500 \mu \mathrm{m}$ to $1 \mathrm{~mm}$ coarse sand $(\Phi: 1-0)$, and $1-2 \mathrm{~mm}=$ very coarse sand ( $\Phi$ : 0 to -1$)$. Additionally, at each sampling date a CTD (Seabird 19) equipped with a Beckman oxygen sensor was used to measure water temperature, salinity and oxygen content at $0.5 \mathrm{~m}$ above the sea floor.

\section{Results}

Oceanographic and sedimentological conditions

Temperature ranged from 14.7 to $20.8^{\circ} \mathrm{C}\left(\Delta 6.1^{\circ} \mathrm{C}\right)$ at $5 \mathrm{~m}$ depth, from 14.1 to $17.3^{\circ} \mathrm{C}\left(\Delta 3.2^{\circ} \mathrm{C}\right)$ at $10 \mathrm{~m}$ and from 13.6 to $17.2^{\circ} \mathrm{C}\left(\Delta 3.6^{\circ} \mathrm{C}\right)$ at $20 \mathrm{~m}$ depth (Fig. 2). Highest temperatures were measured at all three depths during the EN event observed in winter 1997 and summer 1998. Oxygen concentrations ranged between $3.5 \mathrm{ml}$ and $6.7 \mathrm{ml}^{-1}(5 \mathrm{~m})$, $1.6 \mathrm{ml}$ and $5.0 \mathrm{ml}^{-1}(10 \mathrm{~m})$ and $0.7 \mathrm{ml}$ and $3.5 \mathrm{ml} \mathrm{l}^{-1}$ $(20 \mathrm{~m})$ (Fig. 2). The present raw data of temperature and oxygen concentration are given in Rojo et al. (2005a-c). The sediment of the study site is dominated by silt and very fine sand (Table 1).
Community analyses

Crustacean species made up $29 \%$ of the total number of species, polychaete worms $27 \%$, gastropods $25 \%$, bivalves $7 \%$, echinoderms $4 \%$ and others (including Cnidaria, Aschelminthes, Polyplacophora, Nemertea, Plathelminthes, Hemichordata and Chordata) made up $8 \%$ of 88 taxa identified. In terms of abundance, crustaceans contributed $30 \%$ to the fauna, gastropods $24 \%$, polychaete worms $19 \%$, bivalves $18 \%$, echinoderms $5 \%$ and others $<5 \%$. All taxa and their biomasses are listed in Table 2 .

About 38 species inhabited the complete depth range. The gastropod Oliva peruviana, the crustaceans Blepharipoda spinimana, Emerita analoga, Lepidopa chilensis, Synalpheus spinifrons, cumaceans and the chordate Branchiostoma elongatum were only collected at shallow depth. The gastropods Agatoma ordinaria, Crassilabrum crassilabrum, Trigonostoma tuberculosum and the crustacean Callianassa sp. occurred only at $10 \mathrm{~m}$ depth whereas the gastropods Aeneator fontainei, Aesopus aliceae, Calliostoma fonki, Nucella sp., the bivalve Solemya atacama, the polychaete families Chaetopteridae, Eunicidae, Maldanidae and the crustacean Gomeza serrata were only present at $20 \mathrm{~m}$. The SIMPER analyses revealed that gammarids (78\%) and Nephtyidae (11\%) were the dominant species at
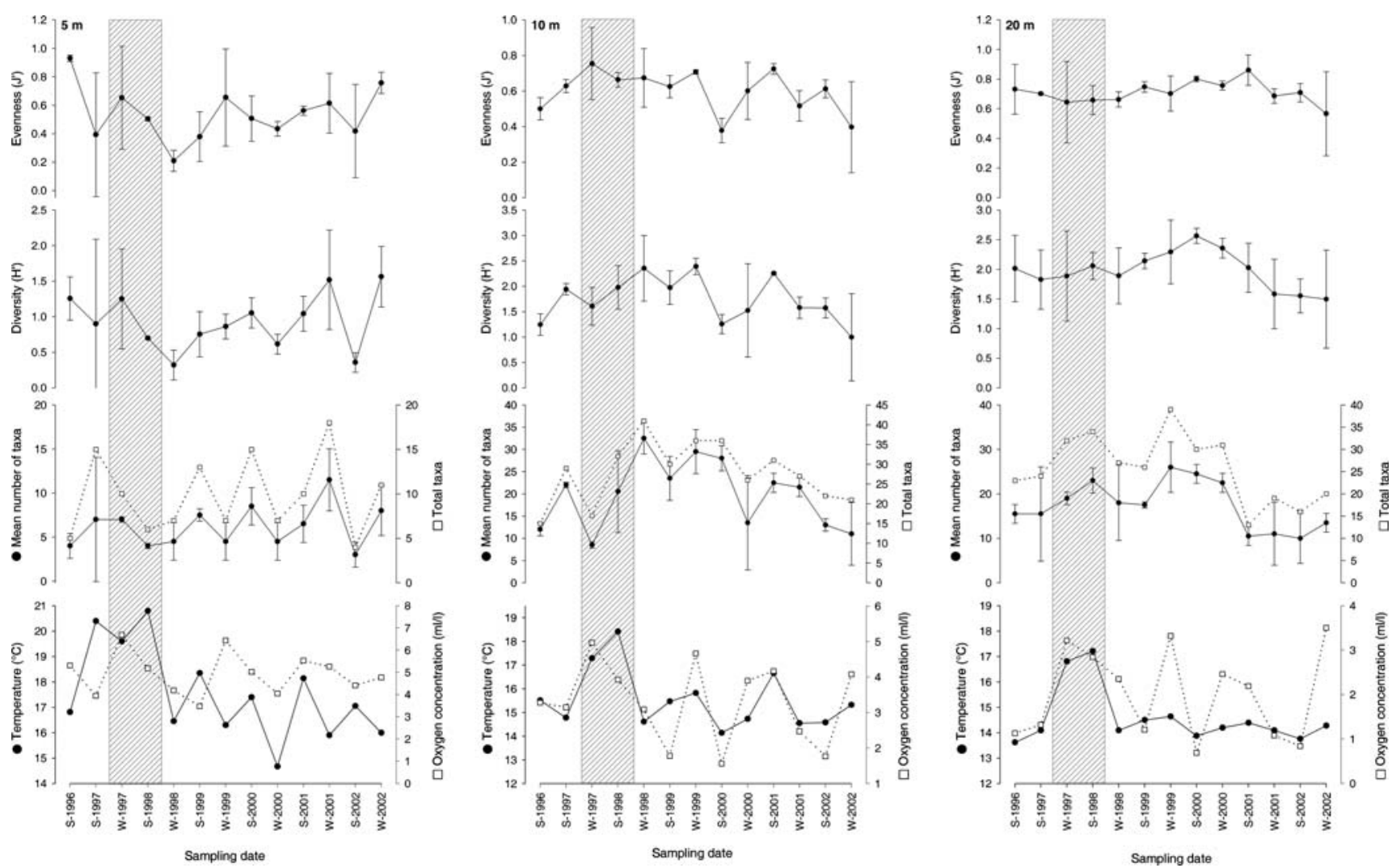

Fig. 2 Temperature and oxygen concentration, mean and total number of taxa, Shannon diversity $\left(\mathrm{H}^{\prime}, \mathrm{Log}\right.$ e) and evenness $\left(\mathrm{J}^{\prime}\right)$ of soft bottom macrobenthos of three different depths (5, 10 and $20 \mathrm{~m}$ ) from Mejillones Bay in northern Chile. Shaded bars indicate El Niño $1997-1998$ 
Table 1 Characterization of the six stations located in Mejillones Bay (raw data available from Rojo and Laudien 2005)

\begin{tabular}{|c|c|c|c|c|c|c|c|c|c|}
\hline \multirow{2}{*}{$\begin{array}{l}\text { Station } \\
\text { number }\end{array}$} & \multirow{2}{*}{$\begin{array}{l}\text { Latitude } \\
\text { (W) }\end{array}$} & \multirow{2}{*}{$\begin{array}{l}\text { Longitude } \\
\text { (S) }\end{array}$} & \multirow{2}{*}{$\begin{array}{l}\text { Water } \\
\text { depth (m) }\end{array}$} & \multicolumn{6}{|c|}{ Sediment fractions $(\%)$} \\
\hline & & & & $\begin{array}{l}\text { Very coarse } \\
\text { sand }(1-2 \mathrm{~mm})\end{array}$ & $\begin{array}{l}\text { Coarse sand } \\
(500 \mu \mathrm{m}-1 \mathrm{~mm})\end{array}$ & $\begin{array}{l}\text { Medium sand } \\
(250-500 \mu \mathrm{m})\end{array}$ & $\begin{array}{l}\text { Fine sand } \\
(125-250 \mu \mathrm{m})\end{array}$ & $\begin{array}{l}\text { Very fine sand } \\
(63-125 \mu \mathrm{m})\end{array}$ & $\begin{array}{l}\text { Silt } \\
(<63 \mu \mathrm{m})\end{array}$ \\
\hline 1 & $23^{\circ} 04^{\prime} 21^{\prime \prime}$ & $70^{\circ} 23^{\prime} 37^{\prime \prime}$ & 5 & 6 & 2 & 2 & 4 & 22 & 64 \\
\hline 2 & $23^{\circ} 04^{\prime} 13^{\prime \prime}$ & $70^{\circ} 23^{\prime} 26^{\prime \prime}$ & 5 & 0 & 1 & 6 & 15 & 50 & 28 \\
\hline 3 & $23^{\circ} 04^{\prime} 18^{\prime \prime}$ & $70^{\circ} 23^{\prime} 42^{\prime \prime}$ & 10 & 2 & 5 & 4 & 5 & 26 & 58 \\
\hline 4 & $23^{\circ} 04^{\prime} 06^{\prime \prime}$ & $70^{\circ} 23^{\prime} 31^{\prime \prime}$ & 10 & 1 & 2 & 4 & 7 & 28 & 58 \\
\hline 5 & $23^{\circ} 04^{\prime} 10^{\prime \prime}$ & $70^{\circ} 23^{\prime} 46^{\prime \prime}$ & 20 & 1 & 2 & 5 & 10 & 41 & 41 \\
\hline 6 & $23^{\circ} 04^{\prime} 02^{\prime \prime}$ & $70^{\circ} 23^{\prime} 34^{\prime \prime}$ & 20 & 1 & 1 & 3 & 5 & 35 & 55 \\
\hline
\end{tabular}

$5 \mathrm{~m}$. The bivalve Aulacomya ater (14\%), the gastropods Mitrella unifasciata (12\%) and Nassarius gayi (7\%), gammarids (11\%), an ophiuroid (8\%) and the decapods Pagurus sp. (6\%) and Euripanopeus crenatus (5\%) dominated the medium depth, while the gastropods Nassarius gayi (18\%), Mitrella unifasciata (10\%) and Crucibulum quiriquinae $(5 \%)$, polychaetes of the family Nereidae $(10 \%)$, Nephtyidae (9\%) and Onuphidae (5\%), and the decapods Pinnixia transversalis and P. valdiviensis (8\%) were the most numerous species at the $20 \mathrm{~m}$ stations.

The total mean faunal abundance was 2,119 individuals $\mathrm{m}^{-2}$, but means for different depths differed significantly (ANOVA, $P<0.02$ ) between 1,113 individuals $\mathrm{m}^{-2}$ at $5 \mathrm{~m}, 3,644$ individuals $\mathrm{m}^{-2}$ at $10 \mathrm{~m}$ and 1,601 individuals $\mathrm{m}^{-2}$ at $20 \mathrm{~m}$. The lowest value was recorded at the shallowest depth with 70 individuals $\mathrm{m}^{-2}$ in summer 1996. At $10 \mathrm{~m}$ the minimal value was recorded in winter 1997, while the abundance at the $20 \mathrm{~m}$ stations was lowest in summer 2001. Significant differences in species richness were detected between $5 \mathrm{~m}$ and the deeper stations (ANOVA, $P<0.0001$ ). The diversity ranged between 0.94 (SD 0.40) at $5 \mathrm{~m}$ and 1.95 (SD 0.33 ) at $20 \mathrm{~m}$; overall diversity was 1.54 (SD 0.58). Highest evenness was estimated at $20 \mathrm{~m}$ $(0.71 \pm 0.07 \mathrm{SD})$ and lowest at $5 \mathrm{~m}(0.54 \pm 0.19 \mathrm{SD})$. Mean formalin wet biomass (shells included) was $73(5 \mathrm{~m})$, $1,519(10 \mathrm{~m})$ and $1,305 \mathrm{~g} \mathrm{~m}^{-2}(20 \mathrm{~m})$, averaging at $966 \mathrm{~g} \mathrm{~m}^{-2}$.

Cluster analysis and a MDS plot of abundance data showed that samples from $5 \mathrm{~m}$ depth mainly differ from the deeper stations (Figs. 3, 4). Furthermore the majority of the $20 \mathrm{~m}$ samples are separated from the $10 \mathrm{~m}$ samples. From the graph it is obvious that the $10 \mathrm{~m}$ sample taken during winter 1997 does not match the 10-m group.

\section{Discussion}

Soft-sediment faunal species composition

The species list presented includes 88 invertebrate taxa, of which $24(27 \%)$ are new records for Mejillones Bay (Table 2). The remaining 64 taxa make up a rather low pro- portion (48\%) of the published benthic macroinvertebrates of the study site, comprising 134 invertebrate taxa (and additional 23 fish and 21 algae species; Zúñiga et al. 1983), the majority of the numerically dominating species has already been reported before (Zúñiga et al. 1983). Both, the analyses of only a single habitat (sand-silt bottom; 5, 10, $20 \mathrm{~m}$ ) and the small sample area may explain differences compared with the previous study of sublittoral benthos taken between 3 and $130 \mathrm{~m}$. However, the main aim of the present study was to reveal spatial and temporal differences in diversity between three depth zones and over a 7-year time period, respectively.

The shallow stations revealed 48 taxa, 69 taxa were found at intermediate depth, and the highest total species number (72) was found at $20 \mathrm{~m}$. Molluscs, crustaceans and polychaete worms dominated the fauna, both in number of species $(28,27$ and 24) and individuals (893, 626 and 372 individuals $\mathrm{m}^{-2}$ ). Echinoderms occurred only in lower numbers (four species, 115 individuals $\mathrm{m}^{-2}$ ) with ophiurids being the most abundant group (in accordance with Zúñiga et al. 1983). While a comparable proportion of molluscs was found by Zúñiga et al. (1983), these authors observed a higher percentage of polychaetes and a lower percentage of crustaceans in their sand stations (molluscs : polychaetes : crustaceans : other invertebrates = 11:8:4:1 as opposed to 10:5:8:2 in the present study). However, our results are consistent with findings of Jaramillo et al. (1998), who analysed the macroinfauna of seven bays off the northern Chilean coast. Their study also revealed that shallower habitats show prevailing abundances of crustaceans while deeper bottoms $(50-65 \mathrm{~m})$ are dominated by polychaetes.

\section{Abundance}

Mean macrobenthos abundance was 2,119 individuals $\mathrm{m}^{-2}$, which is consistent with 2,762 individuals $\mathrm{m}^{-2}$ estimated by Zúñiga et al. (1983) for their 3-20 m stations. The most abundant bivalve found during our long-term survey was the mussel Aulacomya ater with maximum abundances of up to 2,415 individuals $\mathrm{m}^{-2}$ at the $10 \mathrm{~m}$ stations in summer 
Table 2 Total abundance (A, individuals $\mathrm{m}^{-2}$ ) and biomass (B, g AFDM m ${ }^{-2}$ ) between summer $(S) 1996$ and winter $(W) 2002$ at 5, 10 and $20 \mathrm{~m}$ water depth, $H$ herbivorous, $C$ carnivorous, $S$ sedimentivoros, $D$ detritivorous, $O$ omnivorous, $S u$ suspensivorous

\begin{tabular}{|c|c|c|c|c|c|c|c|c|c|c|c|c|}
\hline \multirow[t]{3}{*}{ Taxon } & \multicolumn{2}{|l|}{$\mathrm{S}$} & \multicolumn{2}{|l|}{$\mathrm{W}$} & \multicolumn{2}{|l|}{$\mathrm{S}$} & \multicolumn{2}{|l|}{$\mathrm{W}$} & \multicolumn{2}{|l|}{$\mathrm{S}$} & \multicolumn{2}{|l|}{$\mathrm{W}$} \\
\hline & \multicolumn{2}{|l|}{$5 \mathrm{~m}$} & \multicolumn{2}{|l|}{$5 \mathrm{~m}$} & \multicolumn{2}{|l|}{$10 \mathrm{~m}$} & \multicolumn{2}{|l|}{$10 \mathrm{~m}$} & \multicolumn{2}{|l|}{$20 \mathrm{~m}$} & \multicolumn{2}{|l|}{$20 \mathrm{~m}$} \\
\hline & A & B & A & B & A & B & A & B & A & B & A & B \\
\hline \multicolumn{13}{|l|}{ Cnidaria } \\
\hline Anthothoe chilensis $(\mathrm{Su})$ & - & - & 50 & 10.080 & 160 & 32.256 & 35 & 7.056 & 115 & 23.184 & 10 & 2.016 \\
\hline \multicolumn{13}{|l|}{ Aschelminthes } \\
\hline Nematoda indet. (D) & 15 & ND & 5 & ND & - & - & 40 & ND & 100 & ND & 80 & ND \\
\hline \multicolumn{13}{|l|}{ Polyplacophora } \\
\hline Chiton sp. $(\mathrm{H})$ & 5 & 0.420 & - & - & 90 & 7.560 & 35 & 2.940 & 80 & 6.720 & 170 & 14.280 \\
\hline \multicolumn{13}{|l|}{ Gastropoda } \\
\hline Aeneator fontainei $(\mathrm{C})$ & - & - & - & - & - & - & - & - & - & - & 20 & ND \\
\hline Aesopus aliceae $(\mathrm{S})^{\mathrm{a}}$ & - & - & - & - & - & - & - & - & - & - & 5 & ND \\
\hline Agathotoma ordinaria $(\mathrm{C})^{a}$ & - & - & - & - & 15 & ND & - & - & - & - & - & - \\
\hline Calliostoma fonkii (C) & - & - & - & - & - & - & - & - & 5 & ND & - & - \\
\hline Calyptraea trochiformis $(\mathrm{S})$ & 15 & 7.223 & - & - & 135 & 65.003 & 40 & 19.260 & 20 & 9.630 & 525 & 252.788 \\
\hline Cancellaria buccinoides (C) & 5 & 5.140 & - & - & 10 & 10.279 & - & - & 110 & 113.069 & 35 & 35.977 \\
\hline Crassilabrum crassilabrum $(\mathrm{S})$ & - & - & - & - & 15 & 34.262 & 5 & 11.421 & - & - & - & - \\
\hline Crepidula dilatata $(\mathrm{S})$ & 10 & 24.711 & - & - & 145 & 358.310 & 95 & 234.755 & - & - & 20 & 49.422 \\
\hline Crucibulum quiriquinae (S) & 5 & 12.058 & - & - & 315 & 759.654 & 105 & 253.218 & 695 & $1,676.062$ & 580 & $1,398.728$ \\
\hline Fissurella peruviana $(\mathrm{H})$ & 15 & 17.445 & - & - & 220 & 255.860 & 30 & 34.890 & 5 & 5.815 & 25 & 29.075 \\
\hline Mitra orientalis $(\mathrm{C})$ & 255 & 13.260 & - & - & 1,090 & 56.680 & 985 & 51.220 & 625 & 32.500 & 720 & 37.440 \\
\hline Mitrella unifasciata (C) & 150 & 15.000 & - & - & 3,040 & 304.000 & 2,040 & 204.000 & 930 & 93.000 & 935 & 93.500 \\
\hline Nassarius gayi (C) & 100 & 11.930 & 5 & 0.597 & 825 & 98.423 & 405 & 48.317 & 1,460 & 174.178 & 1,520 & 181.336 \\
\hline Nucella sp. (C) ${ }^{a}$ & - & - & - & - & - & - & - & - & 25 & ND & 15 & ND \\
\hline Oliva peruviana $(\mathrm{C})$ & 30 & 89.559 & 45 & 134.339 & - & - & - & - & - & - & - & - \\
\hline Polinices uber $(\mathrm{C})$ & 10 & 6.483 & 30 & 19.449 & - & - & 5 & 3.242 & - & - & 5 & 3.242 \\
\hline Priene rude $(\mathrm{C})$ & 10 & 38.753 & - & - & 230 & 891.319 & 45 & 174.389 & 230 & 891.319 & 80 & 310.024 \\
\hline Salitra radwini $(\mathrm{C})^{\mathrm{a}}$ & - & - & - & - & 15 & 0.248 & 25 & 0.413 & 80 & 1.320 & 90 & 1.485 \\
\hline Tegula luctuosa $(\mathrm{C})$ & 35 & 46.424 & - & - & 55 & 72.952 & 30 & 39.792 & - & - & 5 & 6.632 \\
\hline Trigonostoma tuberculosum (C) & - & - & - & - & 20 & 201.314 & 10 & 100.657 & - & - & - & - \\
\hline Xanthochorus cassidiformis (C) & 15 & 106.338 & 5 & 35.446 & 15 & 106.338 & 5 & 35.446 & 70 & 496.244 & 10 & 70.892 \\
\hline \multicolumn{13}{|l|}{ Bivalvia } \\
\hline Argopecten purpuratus $(\mathrm{Su})$ & - & - & - & - & 90 & 469.188 & 45 & 234.594 & 10 & 52.132 & 30 & 156.396 \\
\hline Aulacomya ater $(\mathrm{Su})$ & - & - & - & - & 7,005 & $6,702.200$ & 2,460 & $6,883.600$ & 615 & $3,942.000$ & 1,025 & $6,570.200$ \\
\hline Gari solida $(\mathrm{Su})$ & - & - & - & - & 20 & 0.616 & 20 & 0.616 & 10 & 0.308 & 5 & 0.154 \\
\hline Nucula sp. (Su) & 70 & 0.973 & - & - & 2,770 & 38.503 & 525 & 7.298 & 5 & 0.070 & 75 & 1.043 \\
\hline Solemya $($ Petrasma $)$ atacama $(\mathrm{Su})^{a}$ & - & - & - & - & - & - & - & - & 110 & 9.966 & 55 & 4.983 \\
\hline Tagelus dombeii $(\mathrm{Su})$ & 80 & ND & 200 & ND & - & - & 5 & ND & 30 & ND & 5 & ND \\
\hline \multicolumn{13}{|l|}{ Polychaeta } \\
\hline Archiannelida indet. (D) ${ }^{\mathrm{a}}$ & - & - & 45 & ND & - & - & - & - & - & - & 10 & ND \\
\hline Capitellidae indet. (C) & - & - & 5 & 0.007 & 140 & 0.182 & 595 & 0.774 & 90 & 0.117 & 315 & 0.410 \\
\hline Chaetopteridae indet. (S) & - & - & - & - & - & - & - & - & - & - & 10 & 7.048 \\
\hline Cirratulidae indet. (S) & - & - & 5 & 0.095 & 10 & 0.100 & - & - & 50 & 0.050 & 165 & 0.165 \\
\hline Eunicidae indet. (C) ${ }^{a}$ & - & - & - & - & - & - & - & - & 5 & ND & - & - \\
\hline Flabelligeridae indet. (S) ${ }^{\mathrm{a}}$ & - & - & - & - & 5 & ND & 5 & ND & - & - & - & - \\
\hline Glyceridae indet. (C) & 10 & 1.387 & 10 & 1.387 & 5 & 0.694 & 15 & 2.081 & 10 & 1.387 & 15 & 2.081 \\
\hline Goniadidae indet. (C) ${ }^{a}$ & - & - & - & - & - & - & 25 & 0.360 & 20 & 0.288 & 5 & 0.072 \\
\hline
\end{tabular}


Table 2 continued

\begin{tabular}{|c|c|c|c|c|c|c|c|c|c|c|c|c|}
\hline \multirow[t]{3}{*}{ Taxon } & \multicolumn{2}{|l|}{$\mathrm{S}$} & \multicolumn{2}{|l|}{ W } & \multicolumn{2}{|l|}{$\mathrm{S}$} & \multicolumn{2}{|l|}{ W } & \multicolumn{2}{|l|}{$\mathrm{S}$} & \multicolumn{2}{|l|}{$\mathrm{W}$} \\
\hline & \multicolumn{2}{|l|}{$5 \mathrm{~m}$} & \multicolumn{2}{|l|}{$5 \mathrm{~m}$} & \multicolumn{2}{|l|}{$10 \mathrm{~m}$} & \multicolumn{2}{|l|}{$10 \mathrm{~m}$} & \multicolumn{2}{|l|}{$20 \mathrm{~m}$} & \multicolumn{2}{|l|}{$20 \mathrm{~m}$} \\
\hline & A & $\mathrm{B}$ & A & $\mathrm{B}$ & A & B & A & B & A & B & A & B \\
\hline Lumbrineridae indet. (D) & - & - & - & - & 60 & 0.738 & 105 & 1.292 & 45 & 0.554 & 110 & 1.353 \\
\hline Magelonidae indet. (D) & - & - & - & - & 10 & ND & - & - & 10 & ND & 10 & ND \\
\hline Maldanidae indet. (D) & - & - & 5 & 0.089 & - & - & - & - & 5 & 0.089 & 25 & 0.443 \\
\hline Nephtyidae indet. (C) & 95 & 0.371 & 250 & 0.975 & 60 & 0.234 & 320 & 1.248 & 605 & 2.360 & 1,220 & 4.758 \\
\hline Nereidae indet. (C) & - & - & 30 & 0.336 & 65 & 0.728 & 180 & 2.016 & 480 & 5.376 & 425 & 4.760 \\
\hline Onuphidae indet. $(\mathrm{O})$ & 15 & 0.852 & 5 & 0.284 & 485 & 27.548 & 395 & 22.436 & 370 & 21.016 & 385 & 21.868 \\
\hline Opheliidae indet. (D) & 20 & 0.618 & 10 & 0.309 & 45 & 1.391 & 5 & 0.155 & 15 & 0.464 & 5 & 0.155 \\
\hline Orbiniidae indet. (D) & 490 & 1.764 & 45 & 0.162 & 330 & 1.188 & 175 & 0.630 & 165 & 0.594 & 140 & 0.504 \\
\hline Paraonidae indet. (S) & - & - & - & - & 5 & ND & - & - & 230 & ND & 210 & ND \\
\hline Phyllodocidae indet. (C) & - & - & - & - & 5 & ND & - & - & - & - & - & - \\
\hline Polychaeta indet. & 15 & ND & 20 & ND & 10 & ND & 20 & ND & 90 & ND & - & - \\
\hline Polynoidae indet. (C) & 25 & 1.055 & 80 & 3.376 & 265 & 11.183 & 385 & 16.247 & 100 & 4.220 & 140 & 5.908 \\
\hline Sabellidae indet. (C) & 5 & ND & - & - & - & - & - & - & 5 & ND & - & - \\
\hline Spionidae indet. $(\mathrm{Su})$ & 65 & 0.117 & 1,690 & 3.042 & 160 & 0.288 & 215 & 0.387 & 475 & 0.855 & 1,110 & 1.998 \\
\hline Syllidae indet. (C) & 15 & ND & 25 & ND & 5 & ND & 50 & ND & 10 & ND & 120 & ND \\
\hline Terebellidae indet. (S) & - & - & - & - & 85 & 25.951 & 90 & 27.477 & - & - & 5 & 1.527 \\
\hline \multicolumn{13}{|l|}{ Nemertini } \\
\hline Nemertina indet. & - & - & 35 & 0.312 & - & - & 35 & 0.312 & - & - & 100 & 0.890 \\
\hline \multicolumn{13}{|l|}{ Plathelmintes } \\
\hline Turbellaria indet. $(\mathrm{H})^{\mathrm{a}}$ & 2,980 & ND & - & - & - & - & 20 & ND & - & - & 5 & ND \\
\hline \multicolumn{13}{|l|}{ Crustacea } \\
\hline Acanthonyx petiveri $(\mathrm{O})^{\mathrm{a}}$ & - & - & - & - & 35 & 0.473 & 45 & 0.608 & - & - & 5 & 0.068 \\
\hline Alpheus inca $(\mathrm{C})^{\mathrm{a}}$ & 5 & 0.607 & - & - & 45 & 5.459 & 30 & 3.639 & - & - & 25 & 3.033 \\
\hline Betaeus truncatus $(\mathrm{C})$ & - & - & - & - & 100 & 3.760 & 30 & 1.128 & 30 & 1.128 & 15 & 0.564 \\
\hline Blepharipoda spinimana $(\mathrm{Su})^{a}$ & 5 & ND & - & - & - & - & - & - & - & - & - & - \\
\hline Callianassa sp. $(\mathrm{C})^{\mathrm{a}}$ & - & - & - & - & 5 & ND & - & - & - & - & - & - \\
\hline Cancer setosus $(\mathrm{C})$ & - & - & - & - & 40 & 27.724 & 5 & 3.466 & 5 & 3.466 & - & - \\
\hline Caprella sp. (D) & - & - & - & - & 7,690 & 10.766 & - & - & 10 & 0.104 & - & - \\
\hline Cumacea indet. (D) ${ }^{\mathrm{a}}$ & 5 & 0.093 & - & - & - & - & - & - & - & - & - & - \\
\hline Emerita analoga $(\mathrm{Su})^{\text {a }}$ & - & - & 220 & 274.494 & - & - & - & - & - & - & - & - \\
\hline Eurypanopeus crenatus $(\mathrm{C})$ & - & - & - & - & 295 & 26.255 & 285 & 25.365 & 115 & 10.235 & 115 & 10.235 \\
\hline Eurypodius latreillei $(\mathrm{O})$ & - & - & - & - & 10 & 0.406 & 25 & 1.015 & 20 & 0.812 & 20 & 0.812 \\
\hline Gammaridae indet. & 2,780 & 3.336 & 3,995 & 4.794 & 2,105 & 2.526 & 3,250 & 3.900 & 130 & 0.156 & 280 & 0.336 \\
\hline Gomeza serrata $(\mathrm{C})^{\mathrm{a}}$ & - & - & - & - & - & - & - & - & - & - & 5 & ND \\
\hline Hepatus chiliensis $(\mathrm{C})$ & 5 & 0.916 & - & - & 5 & 0.916 & 5 & 0.916 & 10 & 1.832 & 5 & 0.916 \\
\hline Inachoides microrhynchus $(\mathrm{O})$ & - & - & - & - & - & - & 15 & ND & 20 & ND & - & - \\
\hline Latreutes antiborealis $(\mathrm{O})$ & 10 & 0.279 & 5 & 0.140 & - & - & 40 & 1.116 & 5 & 0.140 & 10 & 0.279 \\
\hline Lepidopa chilensis $(\mathrm{Su})^{a}$ & 5 & ND & - & - & - & - & - & - & - & - & - & - \\
\hline Mursia gaudichaudi (C) & - & - & - & - & - & - & 5 & ND & 80 & ND & - & - \\
\hline Pagurus sp. (C) & 40 & 2.128 & 10 & 0.532 & 390 & 20.748 & 600 & 31.920 & 230 & 12.236 & 90 & 4.788 \\
\hline Pilumnoides perlatus (C) & - & - & - & - & 125 & 11.150 & 135 & 12.042 & 25 & 2.230 & 5 & 0.446 \\
\hline $\begin{array}{l}\text { Pinnixa transversalis \& } \\
\text { P. valdiviensis }(\mathrm{O})\end{array}$ & - & - & - & - & 15 & 0.293 & 25 & 0.488 & 325 & 6.338 & 280 & 5.460 \\
\hline Pseudocorystes sicarius $(\mathrm{C})^{\mathrm{a}}$ & 5 & ND & - & - & 10 & ND & - & - & - & - & - & - \\
\hline Pseudosquillopsis lessonii $(\mathrm{C})^{\mathrm{a}}$ & - & - & - & - & - & - & - & - & - & - & 5 & ND \\
\hline
\end{tabular}


Table 2 continued

\begin{tabular}{|c|c|c|c|c|c|c|c|c|c|c|c|c|c|}
\hline \multirow[t]{3}{*}{ Taxon } & \multicolumn{3}{|l|}{$\mathrm{S}$} & \multicolumn{2}{|l|}{$\mathrm{W}$} & \multicolumn{2}{|l|}{$\mathrm{S}$} & \multicolumn{2}{|l|}{ W } & \multicolumn{2}{|l|}{$\mathrm{S}$} & \multicolumn{2}{|l|}{ W } \\
\hline & \multicolumn{3}{|c|}{$5 \mathrm{~m}$} & \multicolumn{2}{|l|}{$5 \mathrm{~m}$} & \multicolumn{2}{|l|}{$10 \mathrm{~m}$} & \multicolumn{2}{|l|}{$10 \mathrm{~m}$} & \multicolumn{2}{|l|}{$20 \mathrm{~m}$} & \multicolumn{2}{|l|}{$20 \mathrm{~m}$} \\
\hline & $\mathrm{A}$ & & B & A & B & A & B & A & B & A & & A & \\
\hline Rhynchocinetes typus $(\mathrm{C})^{\mathrm{a}}$ & & - & - & - & - & 10 & 0.049 & 55 & 0.270 & - & - & 5 & 0.115 \\
\hline Synalpheus spinifrons $(\mathrm{C})^{\mathrm{a}}$ & & - & - & - & - & 15 & ND & - & - & - & - & - & - \\
\hline \multicolumn{14}{|l|}{ Echinodermata } \\
\hline Arbacia spatuligera $(\mathrm{H})$ & & - & - & - & - & 5 & 5.211 & 5 & 5.211 & 20 & 20.842 & - & - \\
\hline Ophiuroidea indet. (D) & & 5 & 0.669 & 5 & 0.669 & 2,150 & 287.455 & 1,925 & 257.373 & 90 & 12.033 & 120 & 16.044 \\
\hline Patiria chilensis $(\mathrm{C})^{\mathrm{a}}$ & & - & - & - & - & 35 & 13.118 & 25 & 9.370 & - & - & 10 & 3.748 \\
\hline Tetrapygus niger $(\mathrm{H})^{a}$ & & - & - & - & - & 75 & 17.505 & 10 & 2.334 & 15 & 3.501 & - & - \\
\hline \multicolumn{14}{|l|}{ Hemichordata } \\
\hline Schizocardium peruvianum & & - & - & 20 & ND & 40 & ND & 5 & ND & - & - & - & - \\
\hline \multicolumn{14}{|l|}{ Chordata } \\
\hline Branchiostoma elongatum $(\mathrm{Su})$ & & 10 & 2.652 & 170 & 45.084 & - & - & - & - & - & - & - & - \\
\hline
\end{tabular}

$N D$ not defined

a new records for Mejillones Bay (cf. Zúñiga et al. 1983; Guzmán et al. 1998)

Fig. 3 Dendrogram resulting from Bray-Curtis similarities of macrozoobenthos from Mejillones Bay, northern Chile, using abundance data. Most samples from the 5-m level are grouped and separated from the rest of the samples ( $30 \%$ level, - - - ). The majority of the $20 \mathrm{~m}$ samples were arranged in one cluster (35\% level, ........), and all $10 \mathrm{~m}$ samples except the one taken in winter 1997 group together including some samples from other depth levels (40\% level)

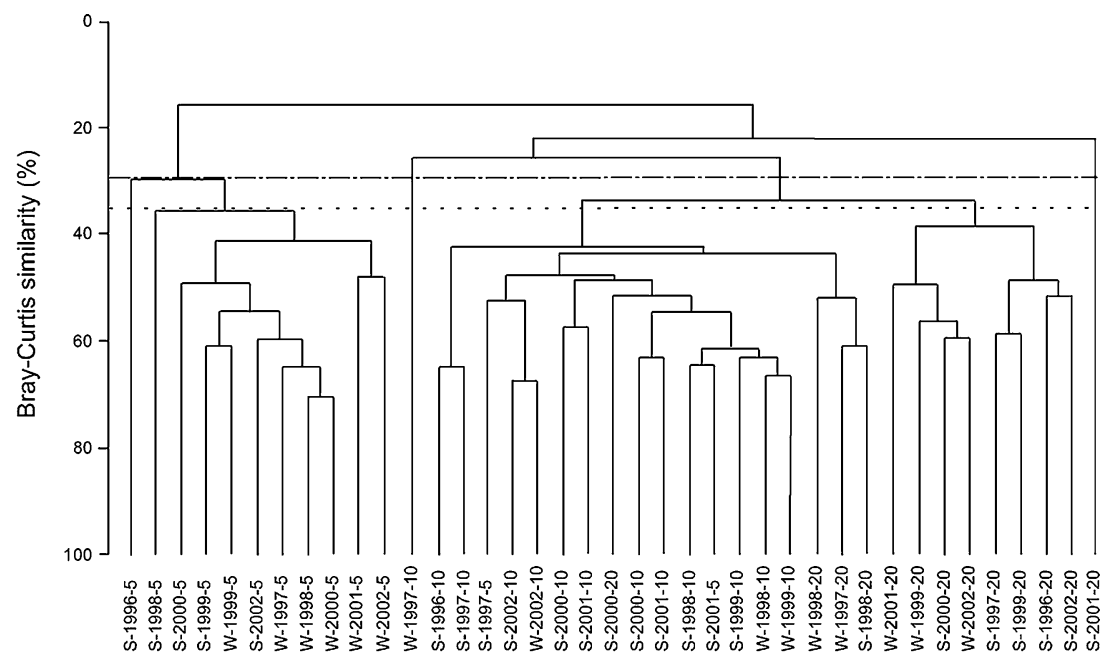

2000, occurring along the whole depth range sampled. It is remarkable that this dominant species was not specifically mentioned in the previous study of Ramorino and Muñiz (1970). Thirteen years later it reached 28.5\% (Zúñiga et al. 1983) of the present mean densities. A possible explanation is that the Pacific Decadal Oscillation shifted to warmer conditions in the 1980s and 1990s (Arntz et al. 2006) and created more favourable conditions for this bivalve off northern Chile. One argument supporting this hypothesis is the fact that commercial exploitation is documented from Antofagasta Bay since 1968, but is registered for Mejillones Bay only since 1984 (SAG 1968-1976; Sernap 1978-1992; Sernapesca 1995-2004). Aulacomya ater is a valuable marine resource for the artisanal fishery and aquaculture in Chile, yielding 4,755 t corresponding to 1.6 Mio US \$ in
2003, and 3,322 t corresponding to 1.1 Mio US \$ in 2004. The respective figures for Mejillones Bay were $217 \mathrm{t}$ equivalent to 72,300 US $\$$ in 2003 , and 123 t equivalent to 40,980 US \$ in 2004 (http://www.fao.org/figis/servlet/ FiRefServlet?ds=species\&fid=3533). Furthermore this mussel provides a habitat for many invertebrate species, a total of 186 species in the case of an Aulacomya ater bed off Pisco, Peru (Tarazona and Arntz 2001).

The second most abundant bivalve was Nucula sp. inhabiting mainly a depth of $10 \mathrm{~m}$. It was also found in high abundances by Ramorino and Muñiz (1970). Their survey revealed that sand and muddy sand habitats were furthermore dominated by the gastropod Priene rude (former Argobuccinum rude) whereas this species ranked only on 6 th position of the most abundant gastropods during the 


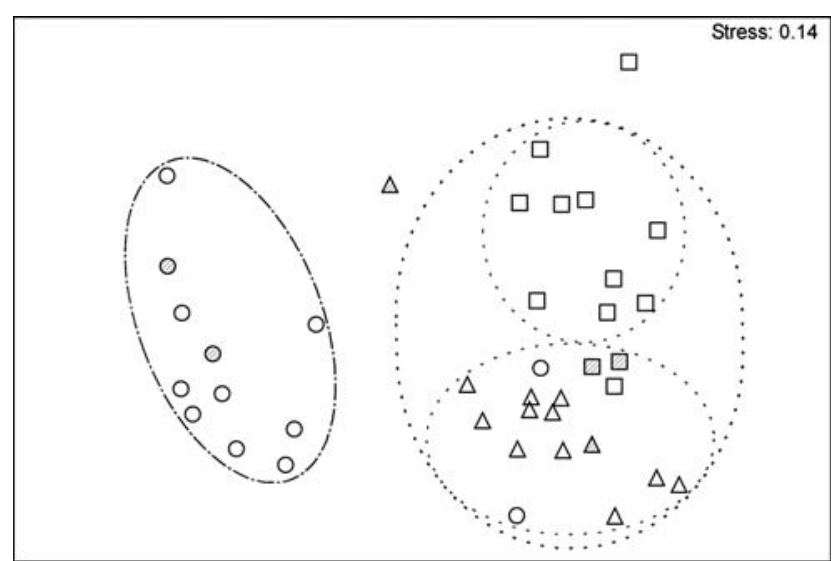

Fig. 4 MDS-plot resulting from classification analysis (Bray-Curtis similarities) of macrozoobenthos from Mejillones Bay, northern Chile, using abundance data. Most samples from the 5-m level (open circle) are more closely related and separated from the rest of the samples (30\% level). Furthermore, the majority of the $20 \mathrm{~m}$ samples (open square) are grouped in one cluster (35\% level) and nearly all $10 \mathrm{~m}$ samples (open triangle) occur in one cluster including some samples from other depth levels. Samples taken during EN 1997-1998 are shaded

present study. Most numerous were Mitrella unifasciata followed by Nassarius gayi, which is in line with the study of Zúñiga et al. (1983).

In the latter and the present survey a hemichordate (Schizocardium peruvianum) occurred only in low abundances. The chordate Branchiostoma elongatum was found regularly during both surveys, in the present study just at $5 \mathrm{~m}$ depth while in the former investigation no depth information was given. In both studies the polychaete family Spionidae was numerically dominant indicating an organically enriched habitat (Heip 1995). Nephtyidae, Orbiniidae and Capitellidae ranked under the six most abundant polychaete taxa. Gammaridae, Caprella sp. and Pagurus sp. were the most abundant crustacean species in both studies.

The present total abundance values are increasing considerably at intermediate depths, which is mainly caused by increasing abundances of the above mentioned bivalves Aulacomya ater and Nucula sp., the gastropod Mitrella unifasciata and ophiuroids. This pattern may reflect the availability of food since primary production is at maximum in a depth of $15 \mathrm{~m}$ (Giraldo et al. 2002). Furthermore, the oxygen concentration measured during the present 7 -year study always exceeded $1 \mathrm{ml}^{-1}$ at the $10 \mathrm{~m}$ stations, whereas lower oxygen concentrations at $20 \mathrm{~m}$ depth might have reduced abundances and limited the distribution of some species (FAO 1975), leading to a virtual absence of macrobenthos at deeper bottoms forming part of the OMZ. Our results are in accordance with findings from the Peruvian Ancón Bay, 1,500 km north of our sampling site: under normal (hypoxic) conditions Tarazona et al. (1988a) estimated maximal values at $15 \mathrm{~m}$ depth $\left(4,000\right.$ individuals $\left.\mathrm{m}^{-2}\right)$. Within the 7 years of our study their maximum value was exceeded only four times at $10 \mathrm{~m}$ depth off Mejillones, with a maximum of 14,440 individuals $\mathrm{m}^{-2}$ (including 7,680 individuals of Caprella sp.), while the maximum value at $20 \mathrm{~m}$ was 2,830 individuals $\mathrm{m}^{-2}$.

\section{Diversity}

Shannon diversity ranged between 0.94 and 1.95, with lower diversity at shallow depths and highest diversity at $20 \mathrm{~m}$. Our values are somewhat lower than the mean of 2.53 calculated from data of Zúñiga et al. (1983) and results published from five different upwelling bays (Table 3). This may be due to the limited area and to the fact that only a single habitat was monitored during the present study.

\section{Biomass}

Mean biomass was $966 \mathrm{~g} \mathrm{~m}^{-2}$ and ranged between $73(5 \mathrm{~m})$ and $1,519 \mathrm{~g} \mathrm{~m}^{-2}(10 \mathrm{~m})$ formalin wet mass (shells included). For the fine sand stations of the same bay Zúñiga et al. (1983) observed a mean of $396 \mathrm{~g} \mathrm{~m}^{-2}$ wet alcohol mass, while Ramorino and Muñiz (1970) estimated $450 \mathrm{~g} \mathrm{~m}^{-2}$ for the coastal zone between 3 and $20 \mathrm{~m}$ and a mean of $569 \mathrm{~g} \mathrm{~m}^{-2}$ at stations with gravel $\left(0.1 \mathrm{~m}^{2}\right.$, Petersen and Smith McIntyre, $0.6 \mathrm{~mm}$ mesh, after $70 \%$ ethanol fixation). Their maximum value of $890.2 \mathrm{~g} \mathrm{~m}^{-2}$ was measured at station No. $31\left(23.05 .1^{\circ} \mathrm{S}, 70.26 .0^{\circ} \mathrm{W}\right)$, which was close to our sampling site $(\sim 3 \mathrm{~km}$ southwest).

At 10 and $20 \mathrm{~m}$ depth our biomass values correspond well with the former studies (Table 4), if the mussel Aulac-
Table 3 Range of diversity $\left(\mathrm{H}^{\prime}\right)$ and evenness $\left(\mathrm{J}^{\prime}\right)$ of five different upwelling bays

\begin{tabular}{lccl}
\hline Site & Diversity $\left(\mathrm{H}^{\prime}\right)$ & Evenness $\left(\mathrm{J}^{\prime}\right)$ & Reference \\
\hline Mejillones (Chile) & $0.94-1.95$ & $0.54-0.71$ & This study \\
Mejillones (Chile) & $1.74-3.06$ & $0.50-0.74$ & Zúñiga et al. (1983) \\
Galicia (Spain) & $2.00-5.00$ & ND & Gomez et al. (2003) \\
Galicia (Spain) & $1.55-4.91$ & $0.55-0.80$ & López-Jamar et al. (1992) \\
Gulf of Cádiz (Spain) & $0.35-2.76$ & $0.18-0.78$ & DelValls et al. (1998) \\
California (USA) & $3.40-3.70$ & $0.40-0.50$ & Bergen et al. (2001) \\
New Jersey (USA) & $1.64-2.41$ & $0.54-0.71$ & Byrnes et al. (2004) \\
\hline
\end{tabular}


Table 4 Percentages of different mayor groups contributing to the total biomass at different areas of Mejillones Bay from three different studies

\begin{tabular}{lllllll}
\hline $\begin{array}{l}\text { Molluscs } \\
(\%)\end{array}$ & $\begin{array}{l}\text { Polychaetes } \\
(\%)\end{array}$ & $\begin{array}{l}\text { Crustaceans } \\
(\%)\end{array}$ & $\begin{array}{l}\text { Other } \\
\text { invertebrates } \\
(\%)\end{array}$ & $\begin{array}{l}\text { Total } \\
\text { biomass } \\
\left(\mathrm{g} \mathrm{m}^{-2}\right)\end{array}$ & $\begin{array}{l}\text { Area depth, } \\
\text { sediment }\end{array}$ & Reference \\
\hline 82.6 & 2.8 & 2.1 & 12.5 & 890.2 & 6 m fine sand & Ramorino and Muñiz (1970) \\
90.0 & 5.6 & 4.3 & 0.1 & 396 & $3-130 \mathrm{~m}$ fine sand & $\begin{array}{l}\text { Zúñiga et al. (1983) } \\
\text { This study }\end{array}$ \\
$98.5(59.5)$ & $0.1(1.7)$ & $1.2(32.1)$ & $0.2(6.7)$ & $73(72)$ & $5 \mathrm{~m}$ fine sand & This study \\
$98.5(83.6)$ & $0.2(2.4)$ & $0.3(3.3)$ & $1.0(10.7)$ & $1,519(474)$ & $10 \mathrm{~m}$ fine sand & This study \\
$98.5(96.0)$ & $0.5(1.4)$ & $0.4(1.0)$ & $0.6(1.6)$ & $1,304(496)$ & $20 \mathrm{~m}$ fine sand & \\
\hline
\end{tabular}

Values of the present study are provided with (and without) the dominating bivalve Aulacomya ater. Biomass in $\mathrm{g} \mathrm{m}^{-2}$ ethanol wet mass in the case of the two former studies, and formalin wet mass (this study)

omya ater is not considered, as this species was no dominant faunistic element in the two earlier investigations (Ramorino and Muñiz 1970; Zúñiga et al. 1983). Thus, the present survey indicates a significant increase in overall biomass resulting from enhanced abundances of Aulacomya ater, which, however, was one order of magnitude lower than that of Peruvian mussel beds (up to 40,000 $\mathrm{g} \mathrm{m}^{-2}$, Tarazona and Arntz 2001).

In agreement with our results, Ramorino and Muñiz (1970) reported that molluscs were the main contributors to overall biomass. Taking into account only their station No. 31 , the percentage of molluscs was $83 \%$ and agrees well with the mean of $89 \%$ of the respective biomass estimated during the present study. Here, the mollusc biomass averaged $893 \mathrm{~g} \mathrm{~m}^{-2}$ formalin wet mass with maximum values of up to $15.9 \mathrm{~kg} \mathrm{~m}^{-2}$ at $10 \mathrm{~m}$ in summer 2000 , which is again mainly allocated to a high biomass of the mussel Aulacomya ater, especially at the 5 and $10 \mathrm{~m}$ stations. Further main contributors were the gastropods Crucibulum quiriquinae (up to $1.1 \mathrm{~kg} \mathrm{~m}^{-2}$, summer 1999, $20 \mathrm{~m}$ ) and Priene rude (up to $290 \mathrm{~g} \mathrm{~m}^{-2}$ in summer 1998 at $20 \mathrm{~m}$ ). Crustaceans made up $2.1 \%$ in the former study but were more prominent during the present investigation (7.6\%). The crustacean biomass was dominated by the decapod Pagurus sp. (17.8 $\mathrm{g} \mathrm{m}^{-2}$ at the $10 \mathrm{~m}$ stations in winter 1998). For the neighbouring Antofagasta Bay (southern bay of the Peninsula Mejillones) Jaramillo et al. (1998) also identified Pagurus villosus as the main contributor to the crustacean biomass. Polychaete worms contributed $0.54 \%$ to the total biomass, which is lower than the mean value of $2.8 \%$ estimated by Ramorino and Muñiz (1970). Terebellidae contributed most to the polychaete biomass reaching up to $26.5 \mathrm{~g} \mathrm{~m}^{-2}$ at the $10 \mathrm{~m}$ stations in winter 2000 , while Onuphidae ranked second accounting for nearly $13.6 \mathrm{~g} \mathrm{~m}^{-2}$ at the $10 \mathrm{~m}$ stations in summer 1998, and for more than $13.1 \mathrm{~g} \mathrm{~m}^{-2}$ at $20 \mathrm{~m}$ in summer 1998 and winter 1999. This is in line with results of Jaramillo et al. (1998) who observed that this polychaete family and to a lesser extent Spiophanes soederstroemi were the major contributors to the secondary production.

\section{Feeding modes}

The fauna was dominated by carnivorous species $(48 \%)$, $15 \%$ were suspensivorous, $13 \%$ sedimentivorous, $11 \%$ detritivorous, $7 \%$ omnivorous and $6 \%$ herbivorous. Dominating carnivores were the gastropods Mitra orientalis, Mitrella unifasciata and Nassarius gayi, the polychaete families Nephtyidae and Nereidae and the crustaceans Gammaridae and Pagurus sp. Prominent suspensivorous species were the bivalves Aulacomya ater and Nucula sp. and the polychaete family Spionidae, while the gastropod Crucibulum quiriquinae dominated the sedimentivorous group. The polychaete families Capitellidae and Orbiniidae, the amphipods Caprella sp. and ophiuroids were the most numerous detritivorous species.

In a study conducted in mid Concepción Bay $(28 \mathrm{~m}$; Central Chile) also carnivorous or omnivorous species were dominating (30\%) the soft sediment fauna. Deposit-feeders contributed $26 \%$, sub-surface deposit-feeders $22 \%$, interface-feeders $13 \%$ and suspension feeders $9 \%$ (Gutiérrez et al. 2000). Differences between the two surveys may be explained by the deeper depth zone sampled in the latter study since there is a gradient in the composition of benthic communities from prevailing abundances of crustaceans and molluscs (predominantly carnivorous and suspensivorous) in shallower habitats, to dominance of polychaetes (mainly sedimentivorous) in deeper zones (Jaramillo et al. 1998), as would be predicted for a highly productive area with high sedimentation rates (Pearson and Rosenberg 1987; Tarazona et al. 1996).

\section{El Niño as a community modulating factor}

According to Ulloa et al. (2001) who analysed time-series data of a coastal location off Antofagasta $\left(23^{\circ} 03^{\prime} \mathrm{S}\right.$, $70^{\circ} 27^{\prime} \mathrm{W}$ ), a first marked deepening of the thermocline and oxycline occurred at the end of April 1997, indicating the arrival of perturbations associated with the start of 1997$1998 \mathrm{EN}$ in the region. Indeed, the $10 \mathrm{~m}$ sample taken during winter 1997 does not match the group of the remaining 
$10 \mathrm{~m}$ samples (Figs. 3, 4). Higher temperatures as a consequence of EN 1997-1998 might have caused this community change. Figure 2 also indicates a significant decrease in species richness at $10 \mathrm{~m}$ depth, which is associated with the onset of EN. The four gastropods Mitrella unifasciata, Nassarius gayi, Nucella sp. and Priene rude, the polychaete families Onuphidae and Polynoidae and the crustacean Eurypanopeus crenatus were especially affected. The population of Aulacomya ater decreased significantly at $10 \mathrm{~m}$ depth, but the abundance of this bivalve increased at $20 \mathrm{~m}$ depth. However, landings of Mejillones Bay dropped from $227 \mathrm{t}$ (yearly mean of first 10 years of exploitation) during and after EN to 74 (1997) and $18 \mathrm{t}$ (1998), respectively, until the previous level was reached again in $2001(246 \mathrm{t})$ (Sernapesca 2001). In line with these findings shallow banks ( $<15 \mathrm{~m}$ ) of Aulacomya ater were destroyed in Peru between Huacho and Pisco during EN 1982-1983 and only deeper populations survived the event (Soenens 1985).

Oxygen saturation was identified to be the ecological master factor modulating sublitoral soft-bottom communities in the Humboldt Current during EN (Tarazona et al. 1988a, b, 1996; Gutiérrez et al. 2000; Peña et al. 2006). The oxygen concentration significantly increased at all depth zones during EN 1997-1998, however, similar high values were also detected during non-EN conditions (Fig. 2). Oxygen concentration of the bottom water at $20 \mathrm{~m}$ depth was never below $0.7 \mathrm{ml}^{-1}$ during the 7 years. Levin et al. (1991) suggested that oxygen concentrations lower than $0.3 \mathrm{ml}^{-1}$ limit macrofaunal community dynamics. Therefore, the observed concentrations may not have exerted inhibitory effects on the benthic fauna of Mejilliones Bay. This is also supported by abundant deposit-feeding polychaetes (Orbiniidae), which are typical of higher successional stages of the community (Tarazona et al. 1988a, 1996; López-Jamar et al. 1992), whereas suspension feeders such as the polychaete Owenia sp. dominated during EN 1992-1993 (Tarazona et al. 1996). The total number of taxa reflected the pattern of oxygen concentration during the first 6 years, and oxygen levels exceeding $2.8 \mathrm{ml} \mathrm{l}^{-1}$ were related to the highest numbers of taxa except for winter 2002.

The oxygen signal of EN is far less clear for the fauna of Mejillones Bay (Fig. 2) than it is off Peru and in deeper living communities of Concepción Bay $(28 \mathrm{~m})$, where Vásquez (1999) observed a significant increase in macrofaunal abundance, biomass and species richness during the 1997$1998 \mathrm{EN}$. In this bay the induced changes followed the clear pattern reported from coastal sediments off Peru during previous EN events (Tarazona et al. 1988a, 1996). Under nonEN conditions the oxygen concentration of the bottom water was low leading to mass mortalities of fauna settled during more favourable conditions (Díaz and Rosenberg 1995; Laudien et al. 2002). During EN oxygen saturation increased significantly $\left(\geq 1 \mathrm{ml} \mathrm{l}^{-1}\right)$, triggering both an extended vertical distribution and a more diverse faunal composition (Gutiérrez et al. 2000). During EN 1982-1983 dissolved oxygen just above the seafloor in Ancón Bay even doubled in relation to average values (Arntz et al. 2006).

This suggests that EN-induced enhanced oxygen levels significantly increase diversity only in those communities, where abundances and species richness are normally limited due to oxygen stress and, thus, unfavourable settling conditions for larvae occur (Arntz et al. 2006; Peña et al. 2006). Oxygen limitation (Levin et al. 1991) explains the low diversity during non-EN years at deeper bottoms (Palma et al. 2005). When physiological tolerance limits are no longer exceeded due to improved oxygen concentrations in the bottom water (Tarazona et al. 1988b; Gutiérrez et al. 2000; Ulloa et al. 2001) previously excluded species may extend their distribution to lower and greater depths ("immigrants" sensu Tarazona et al. 1988a) or proliferate strongly ("opportunists"). Additionally subordinate and carnivorous species may be allowed to re-emerge (Valdivia et al. 2005). This is in line with observations of Tarazona et al. (1996) who stated that very strong or very prolonged EN events may produce favourable conditions only in hypoxic areas, reflected in considerable community changes although the species numbers did never reach those of comparable bays with better oxygenation. Although McPhaden (1999) suggested the 1997-1998 EN as the strongest on record, according to some physical indices, the biological impact off northern Chile was not as catastrophic as generally expected. This does not only hold true for the analysed soft-bottom community, but also for the pelagic ecosystem of the same area (Ulloa et al. 2001). Differences in the impact compared with previous events may be attributed to the distinct seasonal onset (Arntz 2002): while EN 19821983 started in austral spring, just before the reproductive season, EN 1987-1998 commenced in late austral autumn (and thus in the northern hemisphere reproductive season).

Acknowledgments We are grateful to all colleagues participating in the sampling and helping to analyse samples, especially to Rosa Chávez, Fernanda Orellana, Nilda Paredes, Isabel Valdivia and Cecilia Villalobos. We would very much like to thank the crew of RV 'Puri' (University of Antofagasta) for help and assistance at sea. The German BMBF and the Chilean CONICYT provided travel allowance (CONICYT/BMBF 2001-137). This is CENSOR contribution 082. The field work was partially financed by INTERACID Chile Ltd.

\section{References}

Alheit J, Bernal P (1993) Effects of physical and biological changes on the biomass yield of the Humboldt Current ecosystem. In: Sherman K, Alexander LM, Gold BD (eds) Large Marine Ecosystems V: Stress, Migration and Sustainability. American Association for the Advancement of Sciences, Washington, pp 53-68

Arntz WE (2002) The role of El Niño, La Niña and climate change in the Pacific Eastern Boundary Currents: An integrated introductory 
view. In: Salinas S, Urban H-J, Arntz WE (eds) Extended Abstracts of the Symposium "Impacts of El Niño and basin-scale climate change on ecosystems and living resources: A comparison between the California and the Humboldt current systems", Invest Mar 30:81-82

Arntz WE, Fahrbach E (1991) El Niño-climate experiment of nature (in German, Spanish edition in 1996, 312pp). Birkhäuser Verlag, Basel (Switzerland)

Arntz WE, Gallardo VA, Gutiérrez D, Isla E, Levin LA, Mendo J, Neira C, Rowe GT, Tarazona J, Wolff M (2006) El Niño and similar perturbation effects on the benthos of the Humboldt, California, and Benguela Current upwelling ecosystems. Ad Geo 6:243-265

Avendaño Díaz M (1993) Données sur la biologie d'Argopecten purpuratus (Lamarck, 1819), mollusque bivalve du Chili. Ph.D. thesis, Université de Bretagne Occidentale, Brest, France

Avendaño Díaz M, Cantillánez M (1992) Colecta artificial de semilla de Argopecten purpuratus (Lamarck, 1819) en la bahía de Mejillones, Chile, II. Observaciones sobre niveles óptimos de captación. Estud Oceanol 11:39-43

Avendaño Díaz M, Le Pennec M (1997) Intraspecific variation in gametogenesis in two populations of the Chilean mollusc bivalve, Argopecten purpuratus (Lamarck). Aquacult Res 28:175-182

Barber RT, Smith RL (1981) Coastal upwelling ecosystems. In: Longhurst AR (ed) Analysis of Marine Ecosystems. Academic, New York, pp 31-68

Bergen M, Weisberg SB, Smith RW, Cadien DB, Dalkey A, Montagne DE, Stull JK, Velarde RG, Ranasinghe JA (2001) Relationship between depth, sediment, latitude, and the structure of benthic infaunal assemblages on the mainland shelf of southern California. Mar Biol 138:637-647

Brandhorst W (1971) Condiciones oceanográficas estivales frente a la costa de Chile. Rev Biol Mar 14:45-84

Byrnes MR, Hammer RM, Thibaut TD, Snyder DB (2004) Effects of sand mining on physical processes and biological communities offshore New Jersey, USA. J Coast Res 20:25-43

Chávez CP, Riquelme SC (1994) Analisis de calidad bacteriológica en reproductores de Argopecten purpuratus (Lamarck, 1819) para su uso en acuicultura. Rev Latinoam Acuicult (Lima) 43:96-99

Clarke KR, Gorley RN (2001) PRIMER v5: User Manual/Tutorial. PRIMER-E, Plymouth, UK

DelValls TA, Conradi M, Garcia-Adiego E, Forja JM, Gómez-Parra A (1998) Analysis of macrobenthic community structure in relation to different environmental sources of contamination in two littoral ecosystems from the Gulf of Cádiz (SW Spain). Hydrobiologia 385:59-70

Díaz RJ, Rosenberg R (1995) Marine benthic hypoxia: A review of its ecological effects and the behavioural responses of benthic macrofauna. Oceanogr Mar Biol Ann Rev 33:245-303

Escribano R (1998) Population dynamics of Calanus chilensis from northern Chile. Fish Oceanogr 7:245-251

Escribano R, Hidalgo P (2000) Spatial distribution of copepods in the north of the Humboldt Current region off Chile during coastal upwelling. J Mar Biol Assoc UK 80:283-290

Escribano R, Marín V, Hidalgo P, Olivares G (2002) Physical-biological interactions in the pelagic ecosystem of the nearshore zone of the northern Humboldt Current system. In: Castilla JC, Largier J (eds) The Oceanography and Ecology of the Near Shore Bays in Chile. Proceedings of the International Symposium on Linkages and Dynamics of Coastal Systems: Open Coast and Embayments, Ediciones Universidad Católica de Chile, Santiago, pp 145-175

Escribano R, Daneri G, Farias L, Gallardo VA, González HE, Gutiérrez D, Lange CB, Morales CE, Pizarro O, Ulloa O (2004) Biological and chemical consequences of the 1997-1998 El Niño in the Chilean coastal upwelling system: a synthesis. Deep Sea Res II 51(20-21):2389-2411
FAO (1975) Grupo mixto de expertos OCMI/FAO/UNESCO/OMS/ OEA/Naciones Unidas sobre los aspectos científicos de la contaminación de las aquas del mar. GESAMP Informes y Estudios 1

Follegati R (1986) Edad, crecimiento y analisis de curvas polinodales de frecuencia de tallas y pesos en locate Thais chocolata, en la II Region. Source Biota Osorno 1:63

Fonseca T (1989) An overview of the poleward undercurrent and upwelling along the Chilean coast. In: Neshyba SJ, Moores CNK, Smith RL, Barber RT (eds) Poleward Flows along Eastern Ocean Boundaries. Springer, New York, pp 203-228

Fuenzalida R (1992a) Proceso de surgencia en la región norte de Chile, latitudes $30^{\circ} 30^{\prime} \mathrm{S}-21^{\circ} 45^{\prime} \mathrm{S}$. Invest Cient Tecnol Ser Cienc Mar 2:79-103

Fuenzalida R (1992b) Oceanographic and meteorological anamolies during the development of El Niño 1991-1992 at the Chilean coast $\left(20^{\circ} 18^{\prime} \mathrm{S}\right)$. Invest Pesq (Chile) 37:67-72

Gallardo VA (1963) Notas sobre la densidad de la fauna bentónica en el sublitoral del norte de Chile. Gayana (Zool) 10:1-15

Gallardo VA, Palma M, Carrasco FD, Gutiérrez D, Levin LA, Cañete JI (2004) Macrobenthic zonation caused by the oxygen minimum zone on the shelf and slope off central Chile. Deep-Sea Res II 51:2475-2490

Giraldo A, Escribano R, Marín V (2002) Spatial distribution of Calanus chilensis off Mejillones Peninsula (northern Chile): ecological consequences upon coastal upwelling. Mar Ecol Prog Ser 230:225-234

Gomez Gesteira JL, Dauvin JC, Salvande Fraga M (2003) Taxonomic level for assessing oil spill effects on soft-bottom sublittoral benthic communities. Mar Pollut Bull 46:562-572

Gonzalez A, Marín V (1998) Distribution and life cycle of Calanus chilensis and Centropages brachiatus in the Chilean coast: a GIS approach. Mar Ecol Prog Ser 165:109-117

Gutiérrez MJ, Zúñiga Romero O (1976) Cancer setosus Molina in the southern Mejillones Bay (Crustacea, Decapoda, Branchyura). Rev Biol Mar 16:1-25

Gutiérrez D, Gallardo VA, Mayor S, Neira C, Vásquez C, Sellanes J, Rivas M, Soto A, Carrasco F, Baltazar M (2000) Effects of dissolved oxygen and fresh organic matter on the bioturbation potential of macrofauna in sublittoral sediments off Central Chile during the 1997/1998 El Niño. Mar Ecol Prog Ser 202:81-99

Guzmán N, Saá S, Ortlieb L (1998) Descriptive catalogue of nearshore molluscs (Gastropoda and Pelecypoda) from Antofagasta area, $23^{\circ} \mathrm{S}$ (Chile). Estud Oceanol Fac Recur Mar Univ Antofagasta 17:17-86

Hebbeln D, and cruise participants (2001) PUCK: report and preliminary results of R/V Sonne SO 156, Valparaíso (Chile)_-Talcahuano (Chile), March 29-May 14, 2001. Ber Geo Univ Bremen 182

Hebbeln D, Wefer G (2003) Final report for the BMBF project PUCK-SO-156 "Interrelationships between productivity and environmental conditions along the Chilean continental slope". FB Geowiss. Univ Bremen

Heip C (1995) Eutrophication and zoobenthos dynamics. Ophelia 41:113-136

Henríquez R, Olivares A (1980) Ciclo estacional de la cholga Aulacomya ater (Molina 1782) en la Bahía de Mejillones. Arch Biol Med Exp 13:74

Jaramillo E, Carrasco F, Quijon P, Pino M, Contreras H (1998) Distribución y estructura comunitaria de la macroinfauna bentónica en la costa del norte de Chile. Rev Hist Nat 71:459-478

Laudien J, Schiedek D, Brey T, Arntz WE, Pörtner H-O (2002) Survivorship of juvenile surf clams Donax serra (Bivalvia, Donacidae) exposed to severe hypoxia and hydrogen sulphide. J Exp Mar Biol Ecol 271:9-23

Levin LA (2003) Oxygen minimum zone benthos: adaptation and community response to hypoxia. Oceanogr Mar Biol Ann Rev $41: 1-4$ 
Levin LA, Huggett CL, Wishner KF (1991) Control of deep-sea benthic community structure by oxygen and organic-matter gradients in the eastern Pacific Ocean. J Mar Res 49:763-800

López-Jamar E, Cal RM, González G, Hanson RB, Rey J, Santiago G, Tenore KR (1992) Upwelling and outwelling effects on the benthic regime of the continental shelf off Galicia, NW Spain. J Mar Res 50:465-488

Mann KH, Lazier JRN (1991) Dynamics of Marine Ecosystems. Blackwell, Oxford

Marín V, Olivares G (1999) Estacionalidad de la productividad primaria en Bahía Mejillones del sur: una aproximación proceso-funcional. Rev Chil Hist Nat 72:629-641

Marín V, Rodríguez L, Vallejo L, Fuenteseca J, Oyarce E (1993) Efectos de la surgencia costera sobre la productividad primaria primavera de Bahía Mejillones del Sur (Antofagasta, Chile). Rev Chil Hist Nat 66:479-491

Marín VH, Escribano R, Delgado LE, Olivares G, Hidalgo P (2001) Nearshore circulation in a coastal upwelling site off the northern Humboldt Current system. Cont Shelf Res 21:1317-1329

McPhaden MJ (1999) Genesis and evolution of the 1997-98 El Niño. Science 283:950-954

Miranda BO (1975) Crecimiento y estructura poblacional de Thais (Stromanita) chocolata (Duclos, 1832), en la bahía de Mejillones de Sur, Chile. (Mollusca, Gastropoda, Thaididae). Rev Biol Mar Dep Oceanol Univ Chile 15:263-286

Moraga D, Avendaño M, Peña J, Le Pennec M, Tanguy A, Baron J (2001) Genetic and morphological differentiation between two pectinid populations of Argopecten purpuratus from the northern Chilean coast. Estud Oceanol 20:51-60

Morales CE, Blanco JS, Braun M, Reyes H, Silva N (1996) Chlorophyll- $a$ distribution and associated oceanographic conditions in the upwelling region off northern Chile during the winter and spring 1993. Deep-Sea Res 43:267-289

Navea E, Miranda O (1980) Ciclo annual de las conditiones oceanográficas en Mejillones del Sur (Chile). Rev Chil Biol Mar 17:97134

Neira C, Gad G, Arroyo NL, Decraemer W (2001) Glochynema bathyperuvensis sp.n. (Nematoda, Epsilonematidae): a new species from Peruvian bathyal sediments, SE Pacific Ocean, Contr Zool 70:147-159

Pagès F, González HE, Ramón M, Sobarzo M, Gili J-M (2001) Gelatinous zooplankton assemblages associated with water masses in the Humboldt Current System, and potential predatory impact by Bassia bassensis (Siphonophora: Calycophorae). Mar Ecol Prog Ser 210:13-24

Palma M, Quiroga E, Gallardo VA, Arntz W, Gerdes D, Schneider W, Hebbeln D (2005) Macrobenthic animal assemblages of the continental margin off Chile $\left(22\right.$ to $\left.42^{\circ} \mathrm{S}\right)$. J Mar Biol Assoc UK 85:233-245

Peña TS, Johst K, Grimm V, Arntz W, Tarazona J (2006) Disentangling the effects of El Niño on a population of the polychaete $\mathrm{Si}$ gambra bassi in the Bay of Ancón, Peru. Ad Geo 6:161-166

Pearson TH, Rosenberg R (1987) Feast and famine: structuring factors in marine benthic communities. In: Gee JHR, Giller PS (eds) Organization of Communities, Past and Present. Blackwell, Oxford, pp 373-395

Ramorino AM, Muñiz L (1970) Estudio cuantitativo general sobre la fauna del fondo de la Bahía de Mejillones. Rev Biol Mar (Valparaíso) 14:79-93

Robles F, Ulloa A, Pineda J (1975) Analysis of two photographs from ERIS as possible indicators of coastal upwelling in northern Chile. In: International Symposium Coastal Upwelling Proceedings. Coquimbo, Chile, 18-19 November

Rodríguez L, Zárate O, Oyarce E (1986) Produccion primaria del fitoplancton y su relación con la temperatura, oxigeno, nutrientes y salinidad en la Bahía de Mejillones del Sur. Rev Biol Mar 22:75-96
Rodríguez L, Marín V, Farías M, Oyarce E (1991) Identification of an upwelling zone by remote sensing and in situ measurements in Mejillones del Sur Bay (Antofagasta-Chile). Sci Mar 55:467-473

Rojo M, Laudien J (2005) Granulometric composition of a soft sediment habitat in Mejillones Bay (northern Chile), PANGAEA, DOI:10.1594/PANGAEA.308893

Rojo M, Laudien J, Thatje S, Oliva M, Arntz W (2005a) Time series_including El Niño 1997/98 — of temperature and oxygen concentrations at station MSB_1 in Mejillones Bay (northern Chilean Humboldt Current Upwelling system), PANGAEA, DOI:10.1594/PANGAEA.269927

Rojo M, Laudien J, Thatje S, Oliva M, Arntz W (2005b) Time seriesincluding El Niño 1997/98 — of temperature and oxygen concentrations at station MSB_2 in Mejillones Bay (northern Chilean Humboldt Current Upwelling system), PANGAEA, DOI:10.1594/PANGAEA.269928

Rojo M, Laudien J, Thatje S, Oliva M, Arntz W (2005c) Time seriesincluding El Niño 1997/98 - of temperature and oxygen concentrations at station MSB_3 in Mejillones Bay (northern Chilean Humboldt Current Upwelling system), PANGAEA, DOI:10.1594/PANGAEA.269929

SAG (1968-1976) Anuario Estadístico de Pesca Servicio Agricola y Ganadero, Division de Pesca y Caza, República de Chile, Ministerio de Agricultura

Santa Cruz GS (1976) Resultados de algunas experiencias de cultivo de cholgas (Aulacomya ater) en la Bahía de Mejillones. Inst Fomento Pesq (Santiago, Chile) 60

Sernap (1978-1992) Anuario Estadístico de Pesca. República de Chile, Ministerio de Economía, Fomento y Reconstrucción

Sernapesca (1995-2004) Anuario Estadístico de Pesca. Servicio Nacional de Pesca. Ministerio de Economía, Fomento y Reconstrucción de Chile, Valparaíso, Chile

Soenens P (1985) Estúdios preliminares sobre el efecto del fenómeno "El Niño" 1982-1983 en comunidades de Aulacomya ater. In: Arntz WE, Landa A, Tarazona J (eds) "El Niño". Su impacto en la fauna marina. Bol Inst Mar Perú-Callao (special edition), 51-53

Strub PT, Mesías JM, Montecino V, Rutllant J, Salinas S (1998) Coastal ocean circulation off western South America. Coastal segment (6,E). In: Robinson AR, Brink KH (eds) The sea 11. Wiley, New York, pp 273-308

Taraba AA (1959) Perfiles batitermográficos frente a la costa de Chile. In: Proceedings of the international Oceanographic Congress, New York

Tarazona J, Arntz WE (2001) The Peruvian coastal upwelling system. In: Seeliger U, Kjerfve B (eds) Coastal marine ecosystems of Latin America. Springer, Berlin, pp 229-244

Tarazona J, Salzwedel H, Arntz W (1988a) Oscillations of macrobenthos in shallow waters of the Peruvian central coast induced by El Niño 1982-83. J Mar Res 46:593-611

Tarazona J, Salzwedel H, Arntz W (1988b) Positive effects of "El Niño" on macrozoobenthos inhabiting hypoxic areas of the Peruvian upwelling system. Oecologia 76:184-190

Tarazona J, Arntz WE, Canahuire E (1996) Impact of two "El Niño" events of different intensity on the hypoxic soft bottom macrobenthos off the central Peruvian coast. Mar Ecol 17:425-446

Tomicic JJ (1985) Efectos del fenómeno El Niño 1982-83 en las comunidades litorales de la Península Mejillones. Invest Pesq (Chile) 32:209-213

Ulloa O, Escribano R, Hormazabal S, Quiñones RA, González RR, Ramos M (2001) Evolution and biological effects of the 1997-98 El Niño in the upwelling ecosystem off northern Chile. Geophys Res Lett 28:1591-1594

Valdés J, Vargas G, Sifeddine A, Ortlieb L, Guiñez M (2005) Distribution and enrichment evaluation of heavy metals in Mejillones Bay $\left(23^{\circ} \mathrm{S}\right)$, Northern Chile: geochemical and statistical approach. Mar Pollut Bull 50:1558-1568 
Valdivia N, Heidemann A, Thiel M, Molis M, Wahl M (2005) Effects of disturbance on the diversity of hard-bottom macrobenthic communities on the coast of Chile. Mar Ecol Prog Ser 299:4554

Vásquez C (1999) Variación temporal y espacial de la macroinfauna bentónica de Bahía Concepción y plataforma continental adya- cente $\left(36^{\circ} 40^{\prime} \mathrm{S}, 73^{\circ} 02^{\prime} \mathrm{W}\right)$. Marine Biology thesis, Univ. Concepción, Concepción

Zúñiga Romero O, Baeza Kuroki H, Castro Romero R (1983) Análisis de la macrofauna bentónica del sublitoral de la Bahía de Mejillones del Sur. Estud Oceanol Fac Recur Mar Univ Antofagasta 3:41-62 